\title{
Crab Pulsar: Enhanced Optical Emission During Giant Radio Pulses
}

\author{
Susan Collins ${ }^{1}$, Andy Shearer ${ }^{1}$, Ben Stappers ${ }^{2}$, Cesare Barbieri ${ }^{3}$, \\ Giampiero Naletto ${ }^{3}$, Luca Zampieri ${ }^{4}$, Enrico Verroi ${ }^{3}$, \\ and Serena Gradari ${ }^{3}$ \\ ${ }^{1}$ NUI Galway, Ireland, \\ email: susan.collins@nuigalway.ie \\ ${ }^{2}$ Jodrell Bank Center for Astrophysics, UK, \\ ${ }^{3}$ University of Padova, Italy, \\ ${ }^{4}$ INAF Astronomical Observatory of Padova, Italy
}

\begin{abstract}
Although optical pulsar studies have been limited to a few favoured objects, the observation of pulsars at optical wavelengths provides an opportunity to derive a number of important pulsar characteristics, including the energy spectrum of the emitting electrons and the geometry of the emission zone. These parameters will be vital for a comprehensive model of pulsar emission mechanisms. Observations of the Crab pulsar with the high-time-resolution photon-tagging photometer IquEYE show an optical-radio delay of $\sim 178 \mu$ s. Incorporating simultaneous Jodrell Bank radio observations suggested a correlation between giant radio pulses and enhanced optical pulses for this pulsar, thus offering possible evidence for the reprocessing of radio photons.
\end{abstract}

Keywords. pulsars: individual (Crab)

\section{Multiwavelength High-Time-Resolution Astronomy}

Observations of the Crab pulsar with the high-time-resolution photometer IquEYE in December 2009 had concurrent radio observations taken on two nights at Jodrell Bank. The radio data were analysed to find so-called 'giant radio pulses' (GRPs): occasional giant pulses with an intensity of up to 1000 times that of a typical pulse (Lorimer and Kramer, 2005.)

To check our reduction and timing correction pipelines, the optical and GRP data were folded using a radio ephemeris specially fitted over the observing dates. The mainpulse GRPs identified for the night of 2009 December 14 are approximately normally distributed about the nominal peak radio phase (Fig. 1). 663 GRPs were identified above a $6.0 \sigma$ threshold, which also had concurrent optical observations.

\section{Summary}

These results, in combination with those of Shearer et al. (2003), have established a clear link between the optical emission and giant radio pulses of the Crab pulsar (Fig. 2.) The delay of $178 \mu$ s recorded here between the radio and optical peaks (Fig. 3) points to a phase and spatial separation of the order of $2^{\circ}$ and/or 50 kilometres. One possible explanation of the optical emission is the reprocessing of radio photons; see Petrova et al. (2009).

We note however that optical polarisation studies (Słowikowska 2009) show an association between the optical polarisation and the arrival phase of the radio precursor. More observations are needed of the linear and circular polarisation during giant radio pulses, as well as a determination of the optical enhancement as a function of GRP phase. 


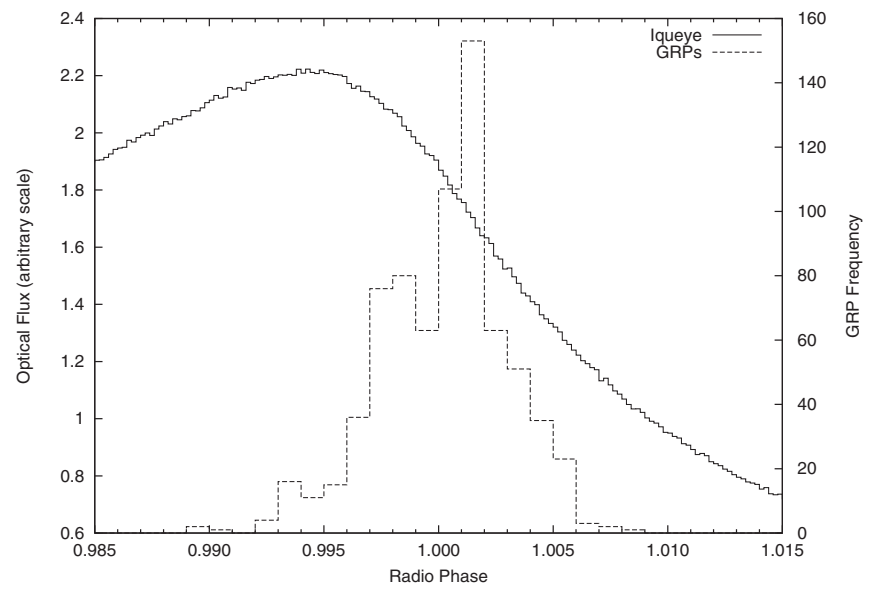

Figure 1. DASHED: Frequency distribution of Crab pulsar Main-Pulse GRPs, with SNR >6.0, observed on 2009 December 14. SOLID: IquEYE optical light curve for the same observing date.

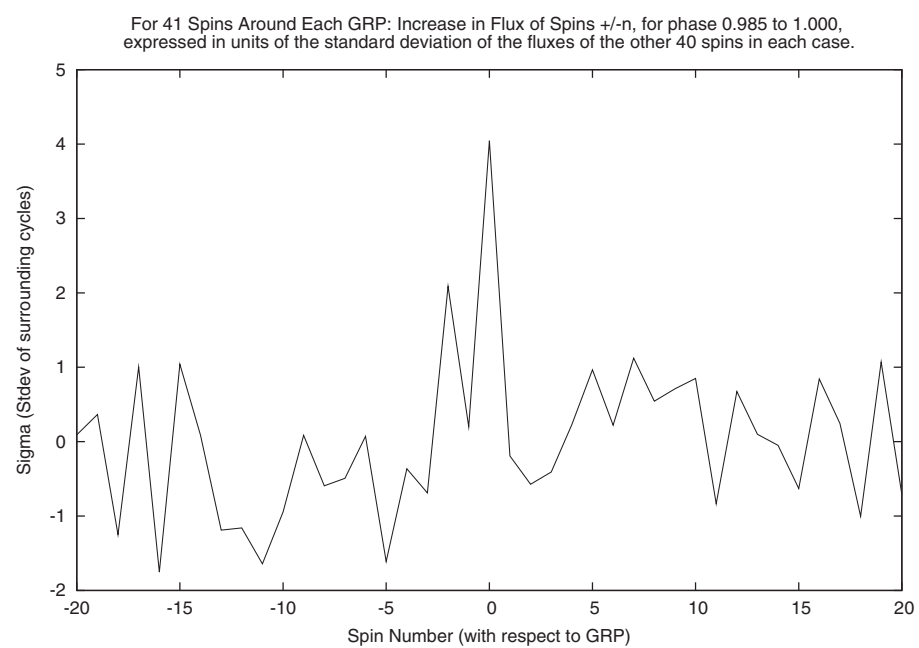

Figure 2. There is a noticeable increase in optical flux at spin0 (which represents the optical photons concurrent with a GRP) up to a $4-\sigma$ level. This correlation between increased optical emission and giant radio-pulse emission is in qualitative agreement with previous findings (Shearer et al., 2003). 


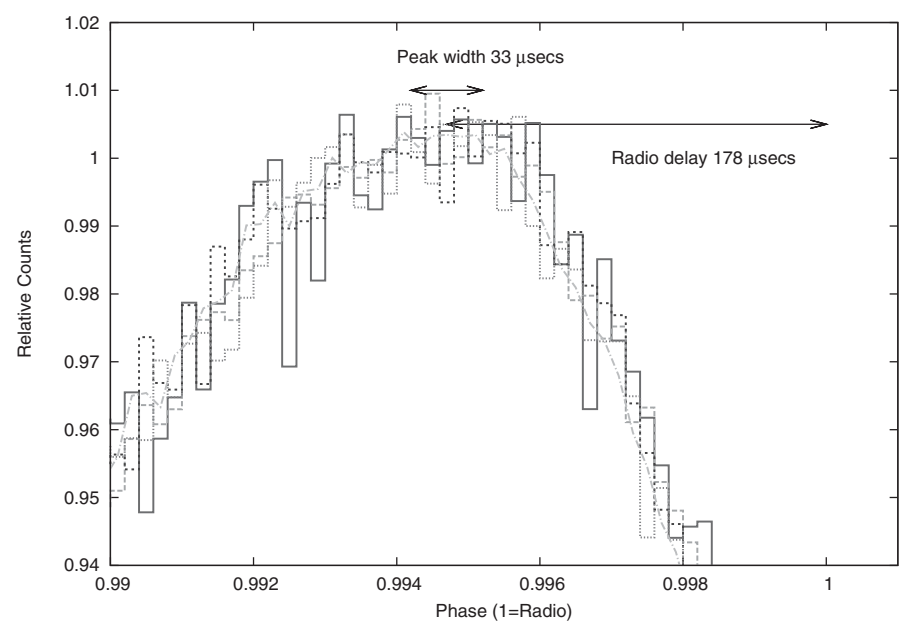

Figure 3. The peak of the optical light curves from 4 nights of data. (For full light curve, see p. 280). There seems to be no night-to-night variation in the average arrival time of the pulses. Furthermore we measured the arrival time of the optical pulse as $\sim 178 \mu$ s before the radio arrival time. That is significantly shorter than recent estimates; for example, Słowikowska et al. (2009) estimated the delay to be $235 \pm 68 \mu$ s. In the combined light over four nights we see a distinct flattening of the peak, with a width of $33 \mu \mathrm{s}$.

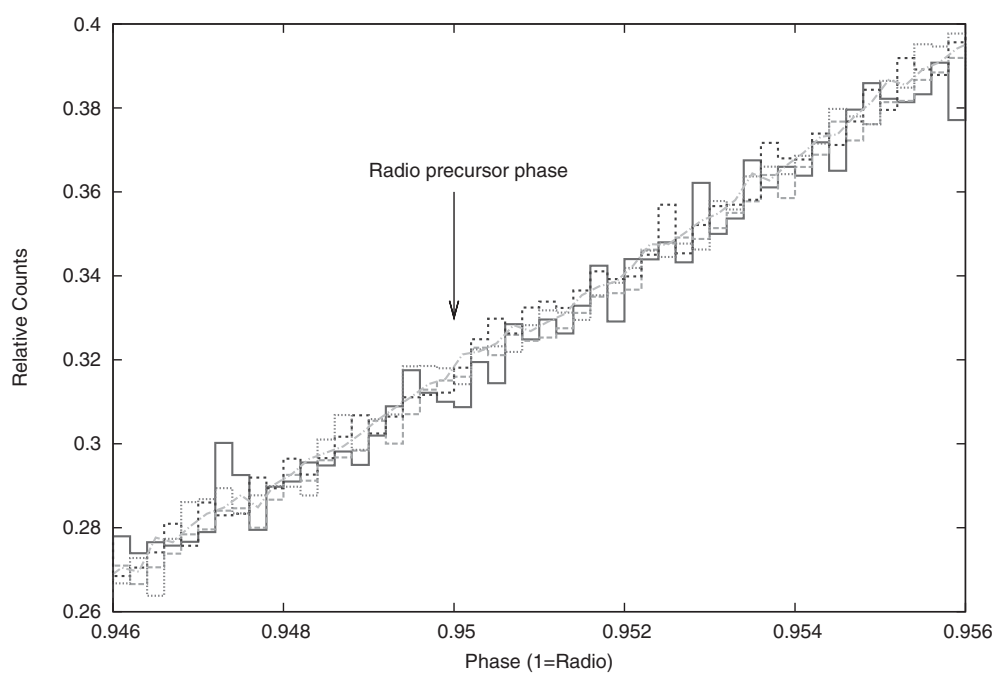

Figure 4. Słowikowska et al. (2009) showed that the linear polarisation of the main optical peak occurs in phase with the radio precursor, at phase .95 . When we look at the optical intensity (Stokes $I$ ) we see no change in the slope of the rising edge. We suggest this is indirect evidence for a shift between linear and circular polarisation as observed by McDonald et al. (2011) and is consistent with a simple synchrotron model for the optical emission.

\section{References}

Lorimer, D. R. \& Kramer, M. 2005, Handbook of Pulsar Astronomy, Cambridge University Press. McDonald, J., et al. 2011, MNRAS, 417 (1) 730

Petrova, S. A. 2009, MNRAS 395, 1723

Shearer, A., et al. 2003, Science 301, 493

Słowikowska, A., Kanbach, G., \& Kramer, M. and Stefanescu, A. 2009, MNRAS 397, (1), 103 